\title{
Unsteady transonic aerodynamics during wing flutter
}

Research article

\author{
Kenichi Saitoh ${ }^{1 *}$, Masato Tamayama ${ }^{1}$ Norio Yoshimoto ${ }^{1}$ Tetsuhiko Ueda ${ }^{2}$ \\ 1 Japan Aerospace Exploration Agency, Tokyo, Japan \\ 2 Nagoya University, Nagoya, Japan
}

Received 03 November 2011 ; accepted 19 March 2012

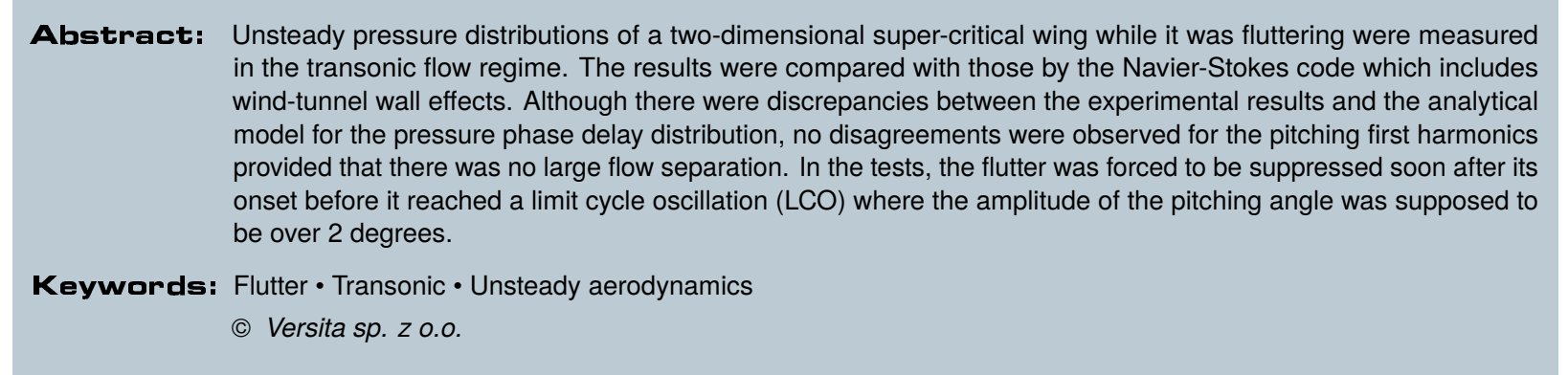

\section{Introduction}

In the transonic regime of flight, the shock wave appears on wing surfaces and affects much on the aeroelastic characteristics as a decrease in the flutter speed due to non-linearity of the aerodynamic forces. Considerable investigation has been pursued at JAXA's transonic flutter wind-tunnel for the non-linear transonic aerodynamics of aeroelastic systems by using two-dimensional wing models [1, 2]. In the earlier stage, the amounts of decrease in flutter critical dynamic pressure and in Mach numbers at the so called transonic dip show disagreement between the experiment and the analysis. There is also a large discrepancy between the analytical model and experimental results even in the case of the static aerodynamic pressure distribution. This incongruity could not be resolved by merely adjusting the flow conditions with Mach numbers or the

${ }^{*}$ E-mail: ksaitoh@chofu.jaxa.jp wing's angles of attack. The reason may be attributed to the wind tunnel walls which restrain the flow. When free flows are compared with and without walls, the disagreement arises when a wind tunnel wing model has relatively large size. Much improvement for flutter critical dynamic pressure as well as the static pressure distribution has been made after the inclusion of wind-tunnel wall effects in the analysis. As a result, the correction in the static pressure distribution improved the accuracy in the prediction of the flutter boundary. However, the detail of the unsteady pressure distribution is not yet clear for the wing in fluttering motion. In the present paper, the unsteady pressure distribution on a wing surface during flutter was measured and discussed.

\section{Wind-tunnel experiments}

The tests were conducted in JAXA's $60 \mathrm{~cm} \sim 60 \mathrm{~cm}$ blowdown Transonic Flutter Wind-tunnel which has plain verti- 




Figure 1. JAXA's Transonic Flutter Wind-tunnel.

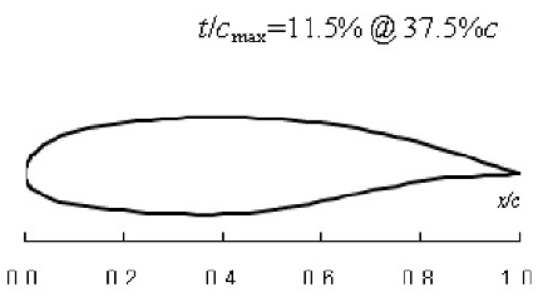

Figure 2. Supercritical airfoil.

cal walls and porous upper and lower walls with a $23.2 \%$ open-hole-ratio. The tunnel is shown in Figure 1.

The airfoil installed has a super-critical shape which assumes a section at $30 \%$ span of the $\mathrm{YXX}$ wing with an $11.5 \%$ thickness ratio. The profile is depicted in Figure 2.

This 2-D wing model made of the 7075 aluminum alloy has $25 \mathrm{~cm}$ in chord length and $59 \mathrm{~cm}$ in span width. Total



Figure 3. Control surface section.

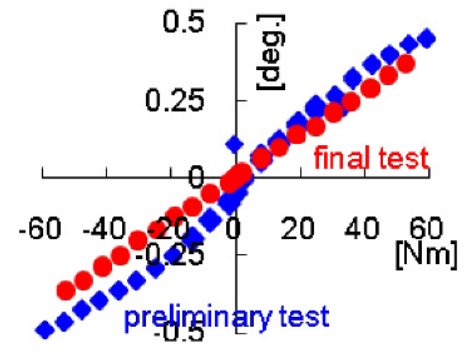

Figure 4. Stiffness test results for pitching motion.

40 holes for static pressure measurements and total 37 unsteady pressure sensors are equipped on the upper and lower surfaces. DSA-3217 of scanivalve was used for measuring static pressure and LQ-47-25 of Kulite transducers for measuring unsteady pressure. The model has also a control surface of which hinge line is located at $80 \%$ of a full chord. In the present experiment, however, it was always tested in the fixed position and the gap between the control surface and the mother wing was sealed with silicone rubber (Figure 3). The wing has also tip plates at both edges sliding along the wall surfaces where a supporting system for the flutter test is furnished. This rigid model is supported by the elastic system which is equipped on the outside of the tunnel-wall and allows for the model to move in both heaving and pitching motions. Displacements of the heaving motion and the pitch angle are measured by four laser triangulators. The heaving stiffness is given by two plate springs located in parallel with the vertical direction. The pitching stiffness is given by a cross spring. The heaving stiffness can be changed by varying the length of the plate and the pitching stiffness can be changed using different kinds of cross springs. The center of gravity and the inertia of rotation are also changeable with additional mass. The pneumatic disk break, which is only for a heaving motion, is equipped to suppress the vibration at flutter. Stiffness tests and adjustments were carried out to eliminate the nonlinearity in the pitching motion that was observed in the preliminary test. These results are shown in Figure 4.

The pitching stiffness was increased after the treatment of this adjustment. The cause of the nonlinearity seems not come from the spring itself but from the alignment of the supporting system. Unbalanced loads at the time of setting may cause the nonlinearity that appears as a hardening type of spring. Therefore, it could also have an effect on the wing to oscillate in the limit cycle oscillation (LCO), which is not our scope in the present investigation. The flutter was always stopped before it reaches LCO where the amplitude of pitching angle might need over 2 degrees. 


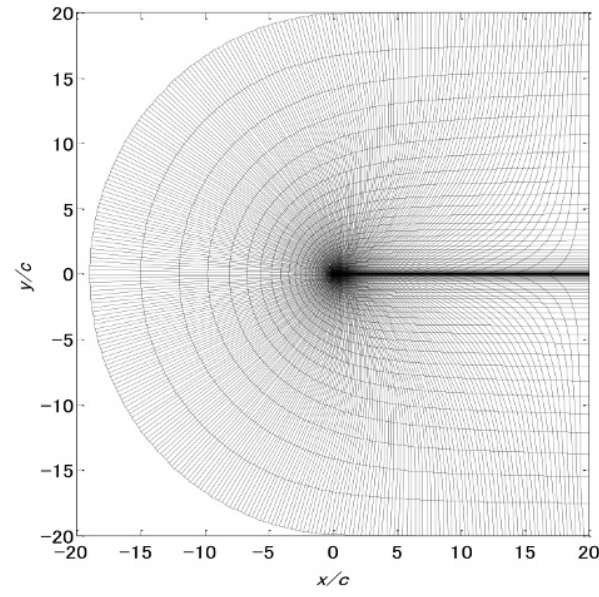

Figure 5. NS Grid (Boundary A).

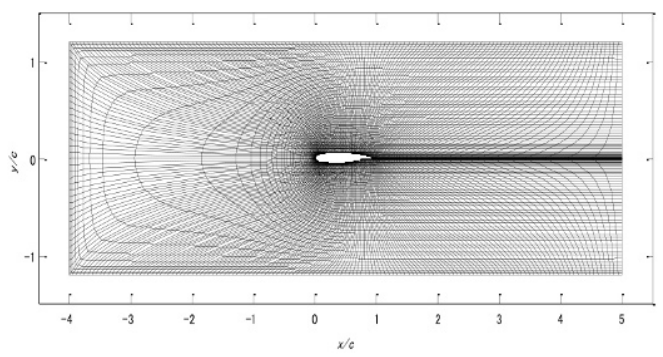

Figure 6. NS Grid for Boundary C.

\section{Flutter analysis}

A two-dimensional NS code developed by Kheirandish et al. [3] with the Baldwin-Lomax turbulence model is used for the CFD computation. A grid system adopted is the structured $C$ type having 313 79 mesh points. It is common to take the computational field boundary sufficiently far from an airfoil by considering the wing chord length as shown in Figure 5 (Boundary A), where the uniform flow condition is applied at the outer boundary. The boundary type used here is corresponding to the reference [1]. The Boundary $A$ has the far field boundaries at $x / c=-19 \sim$ +20 and $y / c=+-20$ whereas the front boundary is semi-circular as shown in Figure 5.

In the present analysis, however, in order to include the wall effects, the other boundary (Boundary C) was used, which can simulate the porous wind-tunnel wall as shown in Figure 6.
This is also the structured C-type and its mesh size is $313 \sim 79$. Outer boundary conditions for the upper and lower walls are given by the following equation:

$$
\frac{w}{U_{\infty}}=\sigma \frac{p-p_{\text {plenum }}}{\rho_{\infty} U_{\infty}^{2}}
$$

where the symbols $U_{\infty}, \rho_{\infty}, p$ and $w$ are the speed, density of uniform flow, pressure, and vertical flow component of disturbed flow, respectively. The symbol $p_{\text {plenum }}$ is a pressure in the plenum chamber. The porosity parameter $\sigma$ assumes 1.0 in the present analysis.

Example calculations for the steady flow with a constant angle of attack and these different boundary grids are shown in Figure 7 and Figure 8.

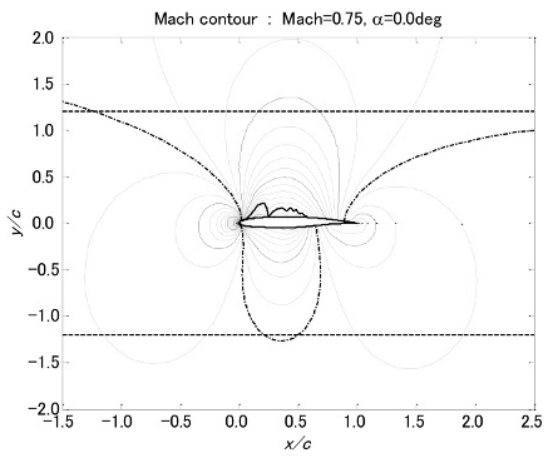

Figure 7. Mach contour with boundary condition A.

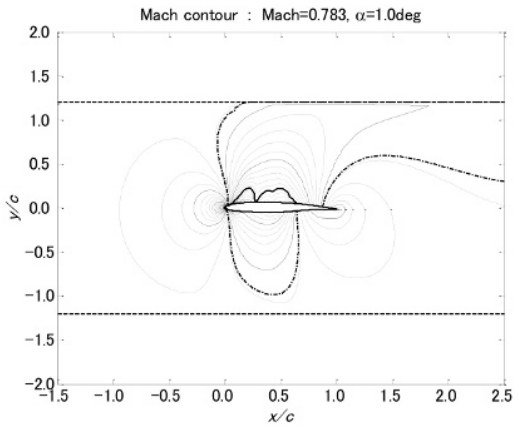

Figure 8. Mach contour with boundary condition C.

It is easy to see that the effect of walls is appreciable in these figures. The matrix equation of an aeroelastic system can be given by:

$$
\left[\begin{array}{cc}
1 & x_{\alpha} \\
x_{\alpha} & r_{\alpha}^{2}
\end{array}\right]\left[\begin{array}{c}
\ddot{h}^{*} \\
\ddot{\alpha}^{*}
\end{array}\right]+\left[\begin{array}{cc}
2 \zeta_{h} \omega_{h}^{*} & 0 \\
0 & r_{\alpha}^{2} 2 \zeta_{\alpha} \omega_{\alpha}^{*}
\end{array}\right]\left[\begin{array}{c}
\dot{\bar{h}}^{*} \\
\dot{\alpha}^{*}
\end{array}\right]+\left[\begin{array}{cc}
\omega_{h}^{* 2} & 0 \\
0 & r_{\alpha}^{2} \omega_{\alpha}^{* 2}
\end{array}\right]\left[\begin{array}{c}
\bar{h} \\
\alpha
\end{array}\right]=\frac{1}{\mu \pi}\left[\begin{array}{c}
-C_{l} \\
2 C_{m}
\end{array}\right]
$$


where the symbols $h, \alpha, x_{\alpha}, r_{\alpha}, \zeta, \omega$ and $\mu$ are the heaving displacement, pitch angle, static unbalance, radius of gyration, damping factor, reduced frequency of vibration and mass ratio, respectively. The suffixes $h$ and $\alpha$ mean the values for the heaving and the pitching motion, respectively. Flutter points were calculated with the aid of the NewtonRaphson method by using the first harmonic components of the lift and the pitching moment in equation (2) [4]. Firstly, a flutter mode is computed by the linear aerodynamic theory which reduces to the eigenvalue problem such as a $p-k$ method [5]. Then, the numerical time marching integration for forced oscillation in the flutter mode follows. Amplitude of the pitching motion is kept constant during this procedure. Secondly, the first harmonic components of the lift and of the pitching moment are calculated from the time history of the CFD simulation. The flutter speed is recalculated by the Newton-Raphson method with these first harmonics. The procedure is iterated until a convergent flutter speed is reached.

\section{Examination for unsteady pres- sure distribution}

The unsteady pressure distribution is examined for the first harmonic components which can be obtained by the following procedure.

In a two-degree-of-freedom system, the displacement of wing surface can be expressed by a combination of pitching and heaving components. By assuming LCO at the flutter, each component is expanded into the Fourier series based on the flutter frequency $\omega$ as follows:

$$
\begin{aligned}
\alpha(t) & =\frac{1}{2} \sum_{l=-\infty}^{\infty} \alpha_{l} e^{i l \omega t} \\
& =\frac{\alpha_{0}}{2}+\sum_{l=1}^{\infty}\left(\alpha_{l, r} \cos (l \omega t)-\alpha_{l, t} \sin (l \omega t)\right) \\
h(t) & =\frac{1}{2} \sum_{l=-\infty}^{\infty} h_{l} e^{i l \omega t} \\
& =\frac{h_{0}}{2}+\sum_{l=1}^{\infty}\left(h_{l, r} \cos (l \omega t)-h_{l, t} \sin (l \omega t)\right)
\end{aligned}
$$

where $\alpha_{l}=\alpha_{l, r}+i \alpha_{l, i}$ and $h_{l}=h_{l, r}+i h_{l, i}$. The flutter mode $q_{f}(t)$ can be given by a combination of pitching and heaving components:

$$
q_{f}(t)=[\alpha(t) \quad h(t)]^{T}
$$

The pressure response at this flutter point is also expanded into Fourier series.

$$
\begin{aligned}
p(t) & =\frac{1}{2} \sum_{l=-\infty}^{\infty} p_{l} e^{i l \omega t} \\
& =\frac{p_{0}}{2}+\sum_{l=1}^{\infty}\left(p_{l, r} \cos (l \omega t)-p_{l, t} \sin (l \omega t)\right)
\end{aligned}
$$

where $p_{l}=p_{l, r}+i p_{l, i}$. By considering the first harmonic, Equation (4) can be rewritten in a complex expression as

$$
q_{f 1}=\left[\begin{array}{ll}
\alpha_{1, r} & h_{1, r}+i h_{1, i}
\end{array}\right]^{T}
$$

where the phase angle is defined as a shift from the pitching oscillation. The first harmonic component of unsteady pressure is also written in the complex form as:

$$
p_{1}=p_{1, r}+i p_{1, i}
$$

where

$$
\begin{aligned}
& p_{1, r}=\frac{1}{\pi} \int_{-\pi / \omega}^{\pi / \omega} p(t) \cos (\omega t) d t \\
& p_{1, i}=-\frac{1}{\pi} \int_{-\pi / \omega}^{\pi / \omega} p(t) \sin (\omega t) d t
\end{aligned}
$$

For unsteady pressure distribution, the normalized pressure coefficient $C_{p}$ is used instead of the pressure itself. It is possible to discuss the aerodynamic forces just for the first harmonic component when it is dominant in the oscillation. The present experimental system has a large mass ratio, which makes the first harmonic component in the oscillation dominant. Unsteady $C_{p}$ can be examined for a pitching oscillation while the heaving component keeps constant in the phase-angle and the amplitude-ratio relative to the pitching motion at flutter. The first harmonic in $C_{p}$ of a pitching motion can be obtained as a form of transfer function $T_{x y}=P_{x y} / P_{x x}$, where $P_{x x}$ and $P_{x y}$ are the power spectrum of unsteady pressure and the cross spectrum between pitching and the pressure, respectively. Although the oscillation in the present experiment was not LCO, the increase in amplitudes was rather gradual at flutter. Therefore, the amplitude can be regarded as constant in a short range of time.

\section{Comparisons of analysis with experiments}

\subsection{Unsteady pressure distribution}

The flutter boundaries obtained in the experiments are listed in Table 1. 
Table 1. Flutter conditions.

\begin{tabular}{cccccccc}
\hline $\begin{array}{c}\text { Test No. } \\
\#\end{array}$ & Mach & $\begin{array}{c}\mathrm{P} 0 \\
\mathrm{kPa}\end{array}$ & $\begin{array}{c}\alpha \text { ini } \\
\text { deg. }\end{array}$ & $\begin{array}{c}\alpha \text { mean } \\
\text { deg. }\end{array}$ & $\begin{array}{c}\Delta \alpha \\
\text { deg. }\end{array}$ & $\begin{array}{c}\Delta \mathrm{h} \\
\mathrm{mm}\end{array}$ & $\begin{array}{c}\text { freq. } \\
\mathrm{Hz}\end{array}$ \\
\hline \hline 09237 & 0,804 & 196 & 1,5 & 0,53 & 0,16 & 0,7 & 22,5 \\
09241 & 0,825 & 177 & 1,5 & 0,53 & 0,13 & 0,5 & 22,0 \\
09242 & 0,777 & 220 & 1,5 & 0,48 & 0,16 & 0,6 & 22,0 \\
09244 & 0,767 & 263 & 1,5 & 0,34 & 0,17 & 0,9 & 23,0 \\
09245 & 0,848 & 246 & 1,5 & 0,15 & 0,08 & 0,8 & 22,0 \\
09254 & 0,817 & 205 & 2,0 & 1,26 & 0,29 & 1,3 & 22,0 \\
\hline
\end{tabular}
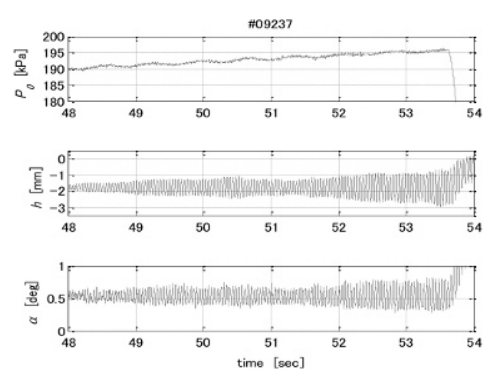

Figure 9. Time history of a total pressure of the flow; $P_{0}$, a heaving displacement; $h$ and a pitch angle; $\alpha$ at flutter (\#09237).
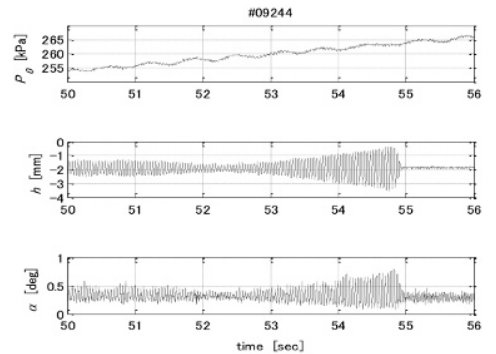

Figure 10. Time history of a total pressure of the flow; $P_{0}$, a heaving displacement; $h$ and a pitch angle; $\alpha$ at flutter (\#09244).

Flutter points were determined while the total pressure of the flow was increasing. Time histories of the total pressure change are shown in Figure 9 and Figure 10 for heaving and pitching, respectively.

The increase in the amplitude at flutter was not fast in general but it depended on the flow condition.

The flutter speeds were also calculated with each corresponding condition.

In order to check the dependency on the time, the pressure distributions at different time points are shown in Figure 11. The unsteady pressure distribution was examined at a certain point during flutter oscillation of which amplitudes were increasing.

In these figures, amplitudes $1 \sim 3$ correspond to the pressure distributions with the averaged pitching angles of $0.08 \mathrm{deg}$, $0.11 \mathrm{deg}$, and $0.16 \mathrm{deg}$, respectively. Those different amplitudes are observed during the increasing of the total
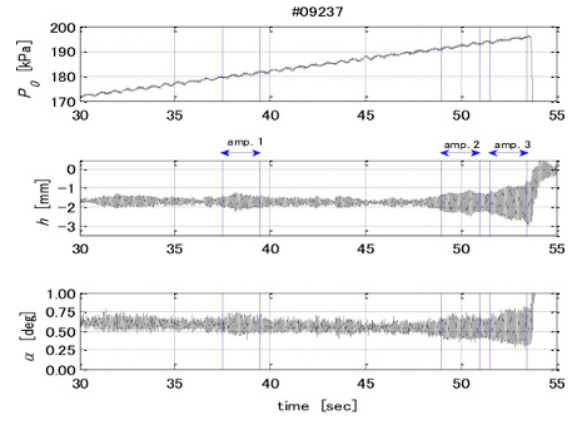

(a) Time history of $P_{0}, h$ and $\alpha$ at flutter (\#09237)

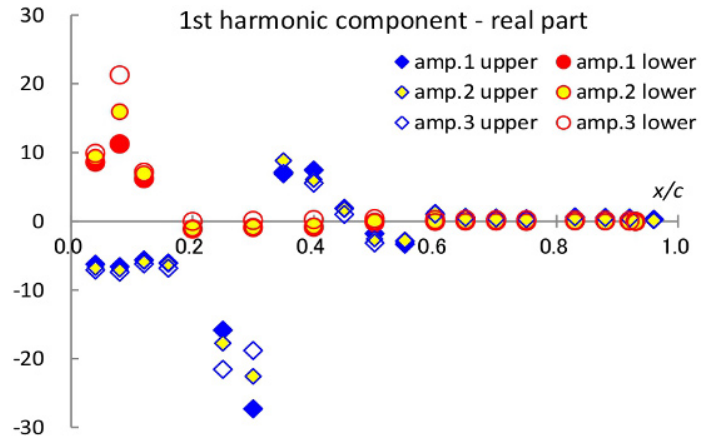

(b) Real part of the pressure distribution

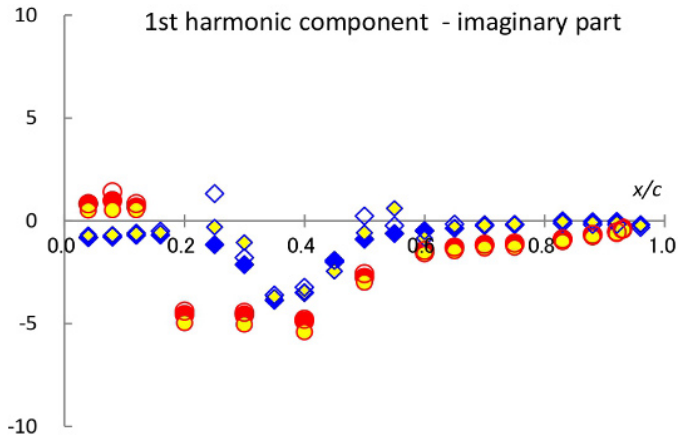

(c) Imaginary part of a pressure distribution

Figure 11. Dependency of pressure distributions on flutter points.

pressure in the wind tunnel before the flutter onset. As the amplitude increases, the peak values on both upper and lower surfaces changes. The point with the maximum amplitude for each flutter is selected for the following discussion of unsteady pressure distributions.

Typical pressure distributions obtained in the experiments and analysis are shown in Figures 12-17.

Symbols $\boldsymbol{\Delta}$ and $\boldsymbol{\nabla}$ indicate the experimental results on the upper and the lower surfaces, respectively. Thick lines and thin lines are analytical results on the upper and the lower surfaces, respectively. Dotted lines in Figure 12 show the computational results with the boundary A. Similar to the correction made in Reference [6], the flow condition is 


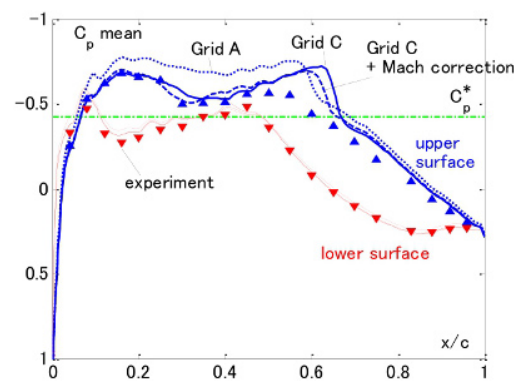

(a) mean pressure distribution

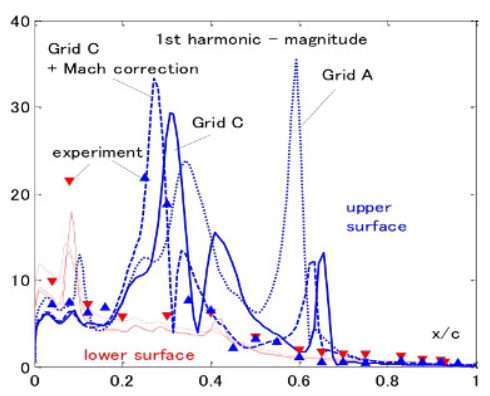

(b) magnitude of the pressure distribution

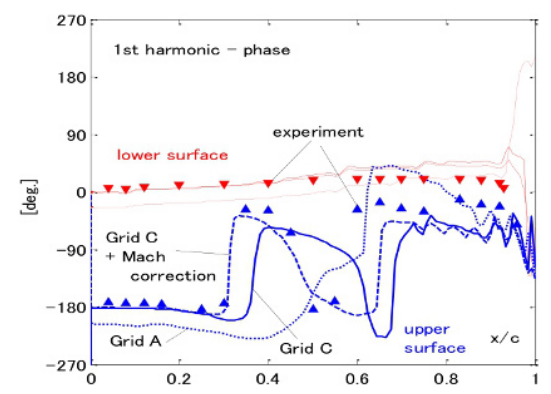

(c) phase of the pressure distribution

Figure 12. Unsteady pressure distribution (\#09237).

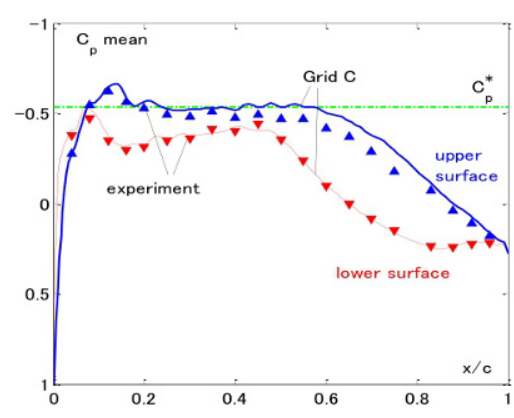

(a) mean pressure distribution

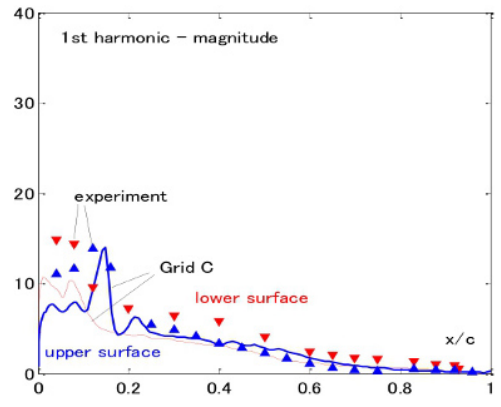

(b) magnitude of the pressure distribution

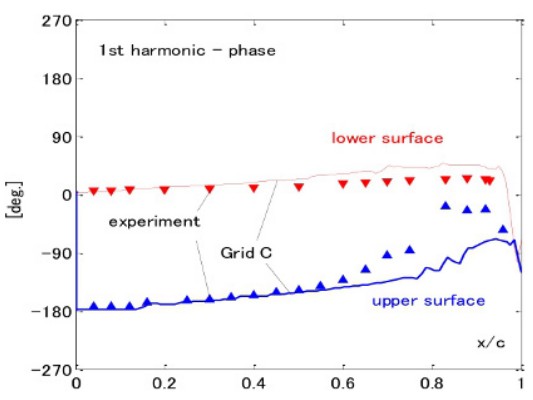

(c) phase of the pressure distribution

Figure 13. Unsteady pressure distribution (\#09244-3).

corrected to obtain better agreement with the experiment, i.e. Mach 0.775 and mean angle of attack $-0.40 \mathrm{deg}$. The computation using the boundary condition $\mathrm{C}$ improves much the agreement with the experiment in both the mean angleof-attack and the first harmonic component of unsteady pressure distribution. Dashed lines in Figure 12 depict the analytical results with Mach 0.799 which is 0.005 lower than the experiment. They are showing better agreement with the experiment. The difference between Mach 0.799 and 0.804 is appreciably seen in the first harmonic component. In most cases, some amount of discrepancy appears around $60 \%$ chord point on the upper surface. It probably needs a more precise boundary condition or a turbulent model to eliminate it. It can be seen from the figure that if the mean pressure distributions agree well to each other then the unsteady pressure also shows good agreement.

It should be pointed out in Figure 13 and Figure 14 that the phase difference between analysis and experiment appears at the lower Mach number near the trailing edge for the lower surface. The reason for this discrepancy is not clear yet. For the amplitude, the difference near the leading edge of the first harmonic component in Figure 13 may be caused by the fast increase in the amplitude at flutter as also shown in Figure 10. At higher Mach numbers than those for the bottom of the transonic dip, it looks quite different in the magnitude of the unsteady component as can be seen in Figure 15 and Figure 16 although the mean pressure distribution in the experiment is rather close to the analysis. It must be due to the flow separation that makes the comparison of unsteady pressure in a harmonic component difficult. The turbulence model with higher accuracy may be required to this flow regime. It can be said, however, that the unsteady pressure distributions can be evaluated with the transfer function of the flutter response to the pressure change unless there exists large flow separation.

\subsection{Flutter (LCO) boundary}

In the experiment conducted in DLR, small amplitude LCO was not observed for a NACA0012 airfoil whereas it occurred on a super-critical NLR7301 airfoil [6]. This may be due to the large unsteady aerodynamic change on the supersonic portion of the super-critical wing [7]. In spite of the fact that the airfoil used in the present experiment is also super-critical one, such small amplitude flutter has 


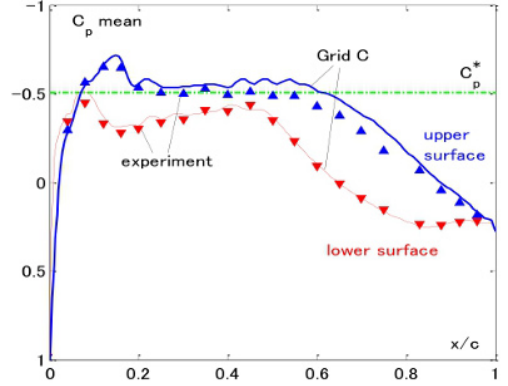

(a) mean pressure distribution

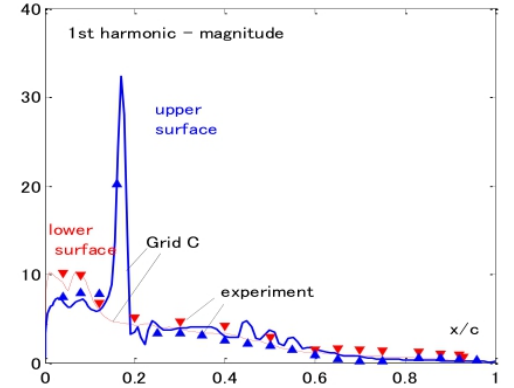

(b) magnitude of the pressure distribution

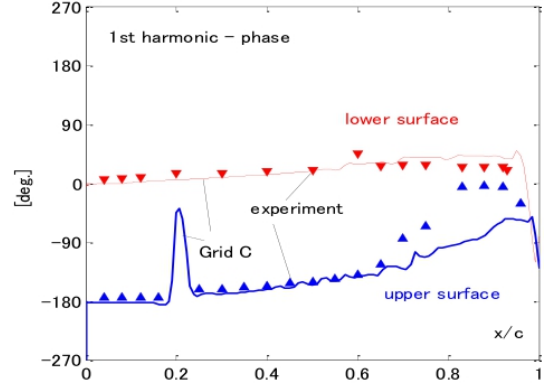

(c) phase of the pressure distribution

Figure 14. Unsteady pressure distribution (\#09242).

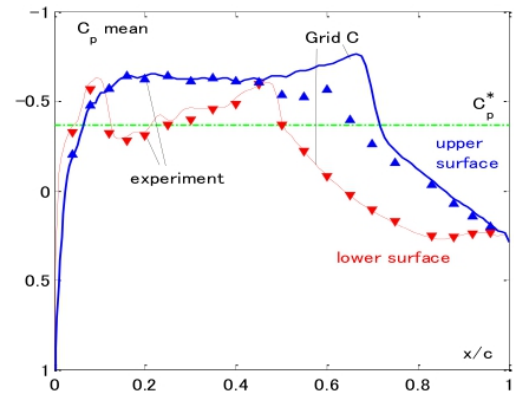

(a) mean pressure distribution

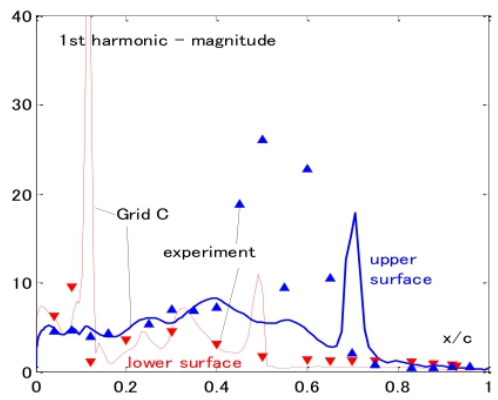

(b) magnitude of the pressure distribution

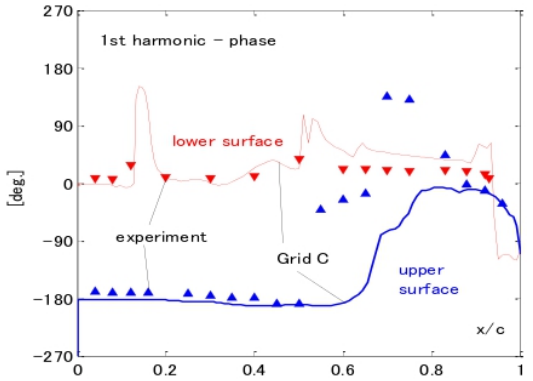

(c) phase of the pressure distribution

Figure 15. Unsteady pressure distribution (\#09241).

not occurred in the tests. Flutter boundaries obtained are shown in Figure 18.

In the experiment, since the incidence of the wing model cannot be changed while the wind is blowing, flutter points were obtained with the same initial angle of attack. A curve indicated as DPM in Figure 18 is a result using the doublet point method (DPM) which is based on the linear aerodynamic theory. The flutter boundary computed by the NS analysis with the boundary $A$ is located closely beneath the DPM result, which shows the transonic dip from the linear theory. The computation has been done for an angleof-attack of 0 deg. With the boundary C, it is computed with the same flow condition and the same flutter mode as measured in the experiment. This boundary is located higher than the experiment, which means unconservative if you think of the actual flutter. However, the shape of the dip and the Mach number for the bottom of the dip is similar to those of the experiments. The structural dynamic model may be corrected to eliminate this discrepancy. In the application of the Newton-Raphson method to obtain flutter boundary with the NS analysis, it kept iterating until the error of the flutter speed becomes less than $2 \%$. Four iterations were sufficient to obtain convergent speed in the most cases.

\section{Conclusion}

Two-dimensional flutter tests were conducted in the transonic flow regime for a supercritical airfoil to clarify the aerodynamic nonlinearity. Unsteady pressure distributions were measured as a transfer function of pressure response due to the pitching component at flutter condition. Numerical computations with the NS code including the wind tunnel wall effects were carried out for the unsteady aerodynamic analysis. Comparisons in the unsteady pressure distributions between experiment and computation thus performed resulted in reasonable agreement. But there is still some discrepancy in the phase angle for the upper surfaces. Accompanied with large flow separation, the evaluation of the unsteady pressure distribution in the first harmonic component becomes difficult.

The NS analysis with wind-tunnel wall effects gives better flutter boundaries than the linear aerodynamic theory and the NS analysis without walls. It was found that the steady state pressure distribution played an important role under conditions of unsteady aerodynamic forces. Certain difference in the flutter boundaries still exists between the experiment and analysis. It may be attributed to the 


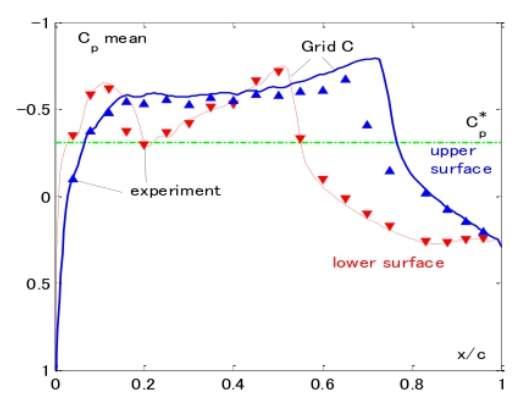

(a) mean pressure distribution

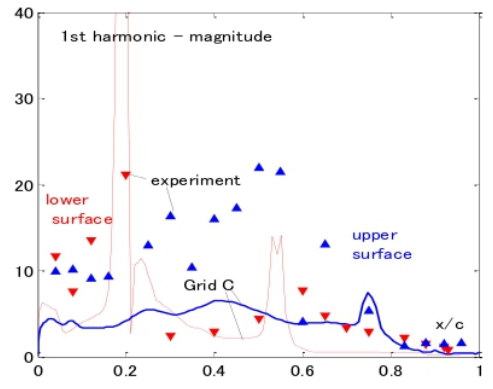

(b) magnitude of the pressure distribution

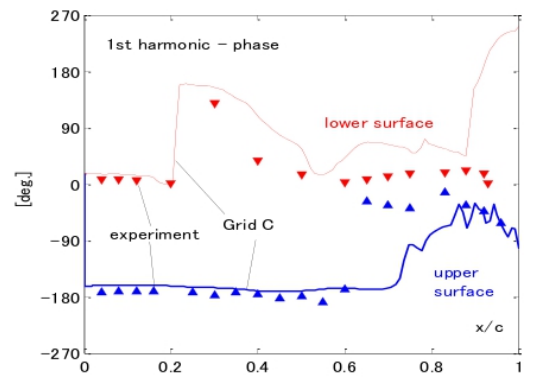

(c) phase of the pressure distribution

Figure 16. Unsteady pressure distribution (\#09245).

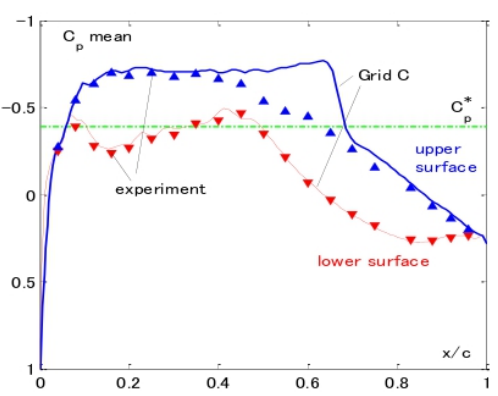

(a) mean pressure distribution

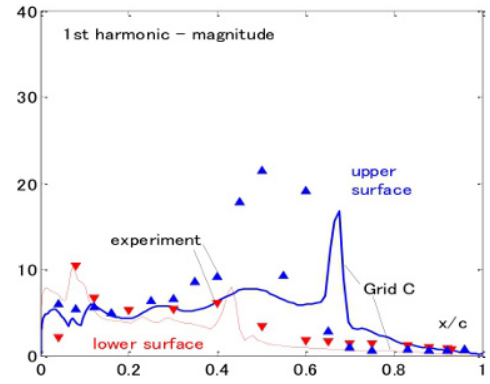

(b) magnitude of the pressure distribution

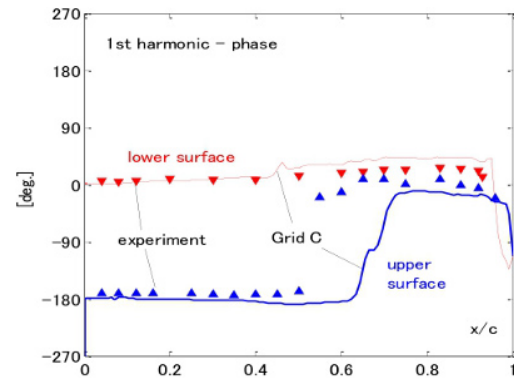

(c) phase of the pressure distribution

Figure 17. Unsteady pressure distribution (09254).

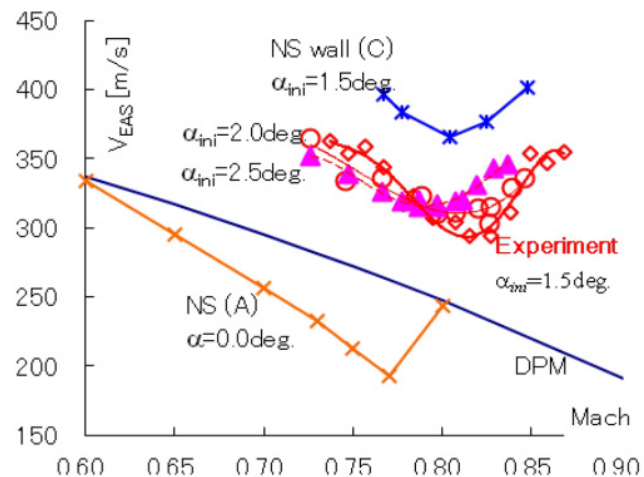

Figure 18. Flutter (LCO) boundaries.

uncertainties of the structural characteristics of a model affected by higher harmonics, the turbulence model for the separation of flow at high Mach numbers above the dip, etc.

\section{References}

[1] Saitoh K., Wind Tunnel Wall Effects on TwoDimensional Flutter tests in transonic flow, The $47^{\text {th }}$ Aircraft Symposium, 2009 (in Japanese)
[2] Saitoh K., Tamayama M., Two-dimensional flutter test of a supercritical wing in transonic regime, IFASD, Seattle, WA, 2009

[3] Kheirandish H.R., Beppu G., Nakamichi J., Numerical flutter simulation of a binary system in transonic region, Aircraft Symposium, Hiroshima, 1995

[4] Thomas J.P., Dowell E.H., Hall K.C., Modeling Viscous Transonic Limit-Cycle Oscillation Behavior Using a Harmonic Balance Approach, J. Aircraft, 2004, 41, 12661274

[5] Hassig, H., An Approximate True Damping Solution of the Flutter Equation by Determinant Iteration, J. Aircraft, 1971, 8, 885-889

[6] Schewe, G., Knipfer, A., Mai, H., Dietz, G.: Experimental and Numerical Investigation of Nonlinear Effects in Transonic Flutter, DLR IB 232-2002J01, 2002

[7] Saitoh K., Nonlinearity of a two-dimensional aeroelastic system in transonic flow, Trans of the Japan Society for Aeronautical and Space Sciences, 2010, 52, 220228

[8] Ueda T., Dowell E., A new solution method for lifting surfaces in subsonic flow, AIAA J., 1982, 3, 348-355 